\title{
Enteropatía por Olmesartán
}

\author{
Olmesartan Enteropathy
}

\author{
Fernández Castro, Isabel; Paz Ferrín, José Manuel: De la Fuente Aguado, Javier \\ Servicio de Medicina Interna. Hospital Policlínico Povisa (Vigo)
}

\begin{abstract}
RESUMEN
El olmesartán es un antagonista de receptores de angiotensina Il comúnmente empleado en el tratamiento de la HTA. Desde el año 2012 se han publicado diversos trabajos alertando de su relación con una enteropatía similar al esprúe celíaco. En nuestro trabajo, comunicamos dos nuevos casos de enteropatía secundaria a tratamiento con Olmesartán y realizamos una revisión de los casos publicados en España y de su fisiopatología y clínica. Como todo efecto adverso atribuido a fármacos, la resolución del cuadro requiere la suspensión del tratamiento con Olmesartán así como tratamiento sintomático del proceso

Palabras clave: olmesartán, enteropatía, esprúe

Keywords: olmesartan, enterophaty, sprue
\end{abstract}

El olmesartán es un antagonista de receptores de angiotensina II comúnmente empleado en el tratamiento de la HTA desde abril de 2002. En agosto de 2012, Rubio-Tapia et al. publicaron una serie de 22 casos a tratamiento con olmesartán que presentaron enteropatía grave y, en julio de 2013, la FDA alertó de la relación entre olmesartán y una enteropatía similar al esprúe celíaco. El objetivo de nuestro trabajo es aportar dos nuevos casos de enteropatía asociada a olmesartán y realizar una revisión de los casos publicados en España. Para ello, efectuamos una revisión bibliográfica en PubMed con las palabras clave "olmesartán" y "enteropatía" desde el año 2012 hasta la actualidad, seleccionando sólo aquéllos publicados por autores en nuestro país.

\section{CASO 1}

Varón de 79 años ingresado en nuestro centro por cuadro de dolor abdominal y diarrea de 6 semanas de evolución, de hasta 20 deposiciones diarias, respetando el sueño, sin moco ni sangre. Refería además pérdida de $10 \mathrm{~kg}$.

El paciente era hipertenso, dislipémico y portador de marcapasos. Estaba a tratamiento con simvastatina $20 \mathrm{mg}$, doxazosina $4 \mathrm{mg}$, pantoprazol $20 \mathrm{mg}$, alopurinol $100 \mathrm{mg}$, olmesartán/hidroclorotiazida 40/12.5 mg. En la exploración física no se detectaron anomalías reseñables, y la analítica no mostró alteraciones. Se le realizaron determinaciones de anticuerpos antigliadina, coprocultivo, parásitos en heces y toxina de Clostridium, todo negativo. Una TAC abdominal fue normal. La colonoscopia mostró mucosa sin úlceras o eritema. En la anatomía patológica se apreció ileítis crónica inespecífica con hiperplasia folicular linfoide sin datos de colitis. Durante el ingreso se suspendió el olmesartán, con progresiva resolución del cuadro hasta desaparecer la diarrea.

\section{CASO 2}

Varón de 78 años que ingresó en nuestro centro por cuadro de dos meses de evolución de hasta 15 deposiciones diarias líquidas, amarillentas, sin productos patológicos ni mejoría con el ayuno y ocasionales náuseas y vómitos. No respetaba el sueño. Refería pérdida de $8 \mathrm{~kg}$ de peso sin hiporexia. No consumo previo de antibióticos. El paciente era hipertenso y dislipémico, a tratamiento con olmesartán e hicroclorotiazida 40/25 mg y atorvastatina $20 \mathrm{mg}$. En Correspondencia: ifernandezc@povisa.es

Como citar este artículo: Fernández Castro I, Paz Ferrín JM, De la Fuente Aguado J.

Enteropatía por Olmesartán. Galicia Clin 2017; 78 (4): 163-164

Recibido: 23/12/2016; Aceptado: 16/01/2017 la exploración física no se detectaron alteraciones relevantes, salvo deshidratación de piel y mucosas. En el hemograma destacaba VSG de $44 \mathrm{~mm} / \mathrm{h}$ y en la bioquímica, un potasio de $2.89 \mathrm{mEq} / \mathrm{l}$. Los anticuerpos antitransglutaminasa y el cultivo, los parásitos y la toxina de Clostridium en heces fueron negativos. La TAC abdominal era normal. La colonoscopia no mostró alteraciones y la anatomía patológica informó de hiperplasia folicular linfoide ileal sin datos de colitis. La gastroscopia con biopsia duodenal fue informada como inflamación crónica en la lámina propia, atrofia focal moderada de vellosidades y linfocitosis intraepitelial CD3 positivas. Durante el ingreso se suspendió el olmesartán y, tras 4 semanas, desapareció completamente la clínica, aumentó 4 kg de peso y normalizó el potasio.

\section{DISCUSIÓN}

El olmesartán es un fármaco ampliamente utilizado en el control de la HTA. Tiene un buen perfil de tolerancia y seguridad, con escasos efectos gastrointestinales ${ }^{1}$. De forma excepcional puede producir una enteropatía grave, que cursa con diarrea importante y pérdida de peso $0^{3-6}$. Como mecanismo fisiopatológico se postula una reacción inmune mediada por células basada en la alteración del mecanismo de regulación intestinal del TGF-B (factor de crecimiento transformante) lo que se traduciría en una alteración en la restitución del epitelio intestinal, deterioro en el crecimiento y la diferenciación de células mesenquimales y una pérdida en la inhibición de linfocitos T y B. Aunque este mecanismo sería similar para todos los ARA-2, hasta la fecha sólo se han descrito casos en asociación con olmesartán. La presencia en las biopsias duodenales de un gran número de CD8 apoyaría esta hipótesis. Los últimos trabajos publicados hacen referencia también a una sobreexpresión de IL-15 que favorece la afluencia de linfocitos T CD8, interfiere en la capacidad supresora y altera la localización de la proteína de unión Z0-1, tal y como ocurre en la respuesta inmune innata de la enfermedad celiaca. En España hay publicados 7 casos, aunque probablemente habrá más debido a un infradiagnóstico o infracomunicación de dicha patología (Tabla 1). En nuestra revisión, apreciamos que la sintomatología aparece más frecuentemente en varones, con 
Tabla 1. Casos de Enteropatía por olmesartán publicados en España

\begin{tabular}{|c|c|c|c|l|}
\hline Sexo & Edad & $\begin{array}{c}\text { Tiempo } \\
\text { tratamiento }\end{array}$ & $\begin{array}{c}\text { Tiempo } \\
\text { clínica }\end{array}$ & Exploración \\
\hline Varón & 78 & 8 años & 2 meses & $\begin{array}{l}\text { Biopsia duodeno: atrofia subtotal de vellosidades, hipeplasia criptas, linfocitosis } \\
\text { intraepitelial y plasmocitosis lámina propia }\end{array}$ \\
\hline Varón & 62 & 12 meses & 3 meses & Biopsia duodeno: linfocitosis intraepitelial y atrofia vellositaria leve \\
\hline Mujer & 82 & 12 meses & 1 mes & Biopsia duodeno: linfocitosis intraepitelial y atrofia vellositaria parcial \\
\hline Varón & 72 & 14 meses & 5 meses & Biopsia duodeno: atrofia vellositaria intensa, nódulos linfoides y linfocitosis intraepitelial. \\
\hline Varón & 75 & 20 meses & Biopsia duodenal: atrofia vellositaria y linfocitosis intraepitelial \\
\hline Varón & 79 & 15 meses & 6 semanas & Biopsia: ilétis crónica inespecífica con hiperplasia folicular linfoide sin datos de colitis \\
\hline Varón & 78 & 18 meses & 2 meses & $\begin{array}{l}\text { Biopsia duodenal: inflamación crónica en la lámina propia, atrofia focal moderada de } \\
\text { vellosidades y linfocitosis intraepitelial CD3 positivas }\end{array}$ \\
\hline Varón & 53 & 26 meses & 6 meses & Biopsia duodenal: atrofia vellositaria (MARSH 3b-3c) \\
\hline Varón & 78 & 36 meses & 4 meses & Biopsia duodenal: atrofia vellositaria \\
\hline
\end{tabular}

Fuente Pubmed.4-8

edades mayores a 70 años y con una duración de tratamiento de al menos un año; sin embargo, en la revisión de la literatura, la aparición de la clínica no parece tener relación con el sexo, la edad o la duración del tratamiento farmacológico. Clínicamente es indistinguible de la enteropatía celíaca, cursando con diarrea, dolor abdominal, pérdida de peso y malabsorción. Recientemente se ha publicado un estudio de cohortes de base poblacional con 20 pacientes de 6 hospitales españoles, en el que hacen referencia a los aspectos antes mencionados y, además, constatan la alta proporción de pacientes que presentan HLA DQ2 y DQ8 positivos (65\%) ${ }^{3}$.

En la anatomía patológica los hallazgos más frecuentes fueron la linfocitosis intraepitelial, atrofia de vellosidades y depósito de colágeno subepitelial. A pesar del alto porcentaje de pacientes que presentan HLA-DQ2 y DQ8 positivos (6 de los 7 casos publicados en España). ${ }^{6-9}$, la diferencia respecto al esprúe celiaco radica en la negatividad de los anticuerpos y en la ausencia de respuesta a la dieta sin gluten. La resolución del cuadro se produce con la suspensión del tratamiento con olmesartán ${ }^{4-9}$. El diagnóstico de certeza de un efecto atribuido a un fármaco exige la desaparición tras la retirada y nueva aparición tras la reintroducción. Sin embargo, cuando este efecto ya está previamente descrito se considera suficiente la resolución del efecto secundario tras la retirada.

La baja incidencia y poco conocimiento de este efecto junto a la concurrencia de la edad, como ocurrió en nuestro caso y los comunicados por otros autores ${ }^{4-8}$, Ileva a realizar múltiples pruebas para descartar otras enfermedades gastrointestinales.
En resumen, ante un paciente con síndrome diarreico crónico sin datos de alarma y a tratamiento con olmesartán, se debe suspender el fármaco y esperar evolución antes de realizar otros procedimientos invasivos.

\section{BIBLIOGRAFÍA}

1. Rubio-Tapia, Herman, Ludvigsson, Kelly, Mangan. Severe spruelike enteropathy associated with olmesartan. Mayo Clin Proc. 2012.

2. DeGaetani, Tennyson, Lebwohl, Lewis, Abu Daya, Arguelles-Grande. Villous atrophy and negative celiacserology: A diagnostic and therapeutic dilema. Am J Gastroente-rol. 2013.

3. US Food and Drug Administration. FDA Drug Safety Communica-tion: FDA approves label changes to include intestinal problems(sprue-like enteropathy) linked to blood pressure medicine olme-sartan medoxomil. http://www.fda.gov/Drugs/DrugSafety/ ucm360122.htm

4. Marietta, Nadeau, Cartee, Singh, Rishi, Choung, Wu, Rubio-Tapia, Murray. Immunopathogenesis of olmesartan-associated enteropathy Aliment Pharmacol Ther. 2015.

5. Esteve, María. Potential coeliac disease markers and autoimmunity in olmesartan induced enteropathy: A population-based study. Dig Liver Dis. 2016.

6. Ulla-Rocha, Lopez-Pineiroy, Puga-Gimenez. Insuficiencia renal aguda secundaria a diarrea severa porenteropatía esprue-like asociada a olmesartán. Gastroenterología y Hepatología. 2014.

7. Muñoz-Muñoz ${ }^{1}$, López-Vivancos, Huaman, García-Cors. Sprue-like enteropathy due to olmesartan. Revista Española de Enfermedades Digestivas 2015.

8. Fiorucci, Puxeddu' ${ }^{1}$, Colella Reboldi, Villanacci, Bassotti. Severe spruelike enteropathy due to olmesartan Revista española de Enfermedades Digestivas. 2014. 